\title{
Use of Electronic Health Records on Days Off: Comparing Physicians to Other EHR Users
}

J Gen Intern Med 36(4):1140-3

DOI: $10.1007 /$ s11606-020-06078-5

(C) Society of General Internal Medicine 2020

\section{INTRODUCTION}

Multiple studies indicate that the US health care system's transition to electronic health records (EHRs) has increased physicians' data entry and documentation burden. ${ }^{1-4}$ This increased time spent on EHR work, particularly during evenings or days off, has been correlated with physician burnout and job dissatisfaction. ${ }^{5,}{ }^{6}$ However, physicians are not the only users of EHRs, and because prior studies do not include the experiences of other EHR users, it remains unclear whether the burden of EHR documentation during off hours uniquely affects physicians or also burdens other staff. Our study sought to explore the extent of off-hour EHR use, comparing physicians to other clinical EHR users.

\section{METHODS}

We repeatedly surveyed EHR end-users in a large safety net hospital system over 2 years. The survey was conducted during a transition to a commercial EHR system, and was administered once prior to the transition and five times following the EHR implementation. The health system includes a 336-bed hospital, a ten-site federally qualified health center, and specialty care practices. We emailed survey invitations to all EHR users at each wave, and accounted for users who joined or left the organization since the last survey. A total of 6593 users had the opportunity to respond to at least one survey wave. Five hundred eighty-three physicians and 2603 other clinical EHR users responded (48.3\% response rate), with many users responding to multiple waves of the survey. In each response, EHR users reported the frequency with which they entered data into the EHR during four scenarios: (1) clinical workdays - in patient rooms with the patient; (2) clinical workdays - between patients; (3) clinical workdays - evenings or nights; and (4) during days off. We calculated the percentage of respondents at each survey time point reporting frequent use, defined as reporting

Received February 21, 2020

Accepted July 17, 2020

Published online August 3, 2020 use "Often" or "All the time," in each scenario. Other response options in the 5-point Likert scale were "Never," "Rarely," or "Sometimes." To explore the unique burden on physicians, we used logistic regression to estimate the relationship between frequent use and role in each scenario, comparing physicians to nurses, physician assistants, nurse practitioners, and other clinical EHR users (e.g., respiratory therapists, behavioral health specialists). Nurses were the reference role, and we adjusted for survey wave, used user-clustered standard errors, and computed average marginal effects (AME).

\section{RESULTS}

Twenty-five percent of physicians reported frequent EHR use on days off, compared with $11 \%$ of NPs and PAs, $5 \%$ of nurses, and $4 \%$ of other clinical users (Fig. 1). Fifty percent of physicians reported frequent EHR workday evening EHR use compared with $41 \%$ of nurses, $35 \%$ of NPs and PAs, and $23 \%$ of other clinical users. In our logistic regression (Fig. 2), physicians' probability of reporting frequent EHR use on days off was 20 percentage points higher than nurses $(\mathrm{AME}=0.20, p<0.001)$. Physicians were 7 percentage points more likely than nurses to report frequent evening use $(\mathrm{AME}=0.07, p=0.008)$ and 11 percentage points more likely to report frequent use between patients $(\mathrm{AME}=0.11, p<0.001)$. In contrast, physicians were less likely to report frequent use during visits $(\mathrm{AME}=-0.18, p<0.001)$. Sensitivity analyses including only post-transition survey waves illustrated similar findings.

\section{DISCUSSION}

Substantial proportions of physicians reported frequent EHR use in the evening and on days off, and at greater rates than other clinicians. Given that EHR use on days off contributes to physician burnout and dissatisfaction, ${ }^{5}$ these findings suggest health systems and technology vendors need to identify approaches to reduce physician documentation burden. Moreover, results held over 2 years; thus, the EHR changeover was unlikely to be the driver of differences between physicians and other users.

Our study has several limitations. First, while results are consistent over time, we are unable to control for unobserved 

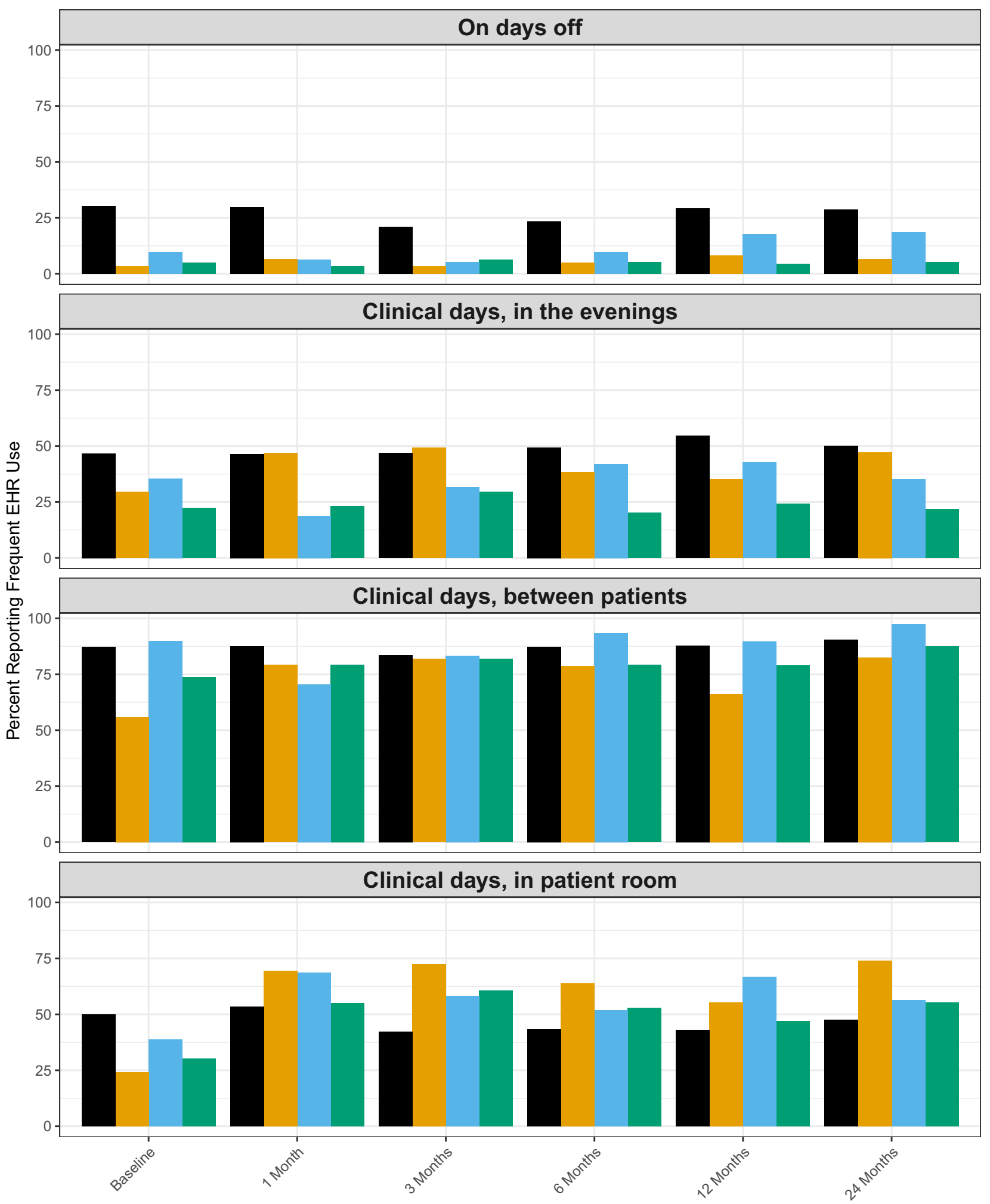

$\begin{array}{lll}\text { Physicians Nurses } \quad \text { NPs \& PAs } & \begin{array}{l}\text { Other Clinical } \\ \text { EHR Users }\end{array}\end{array}$

Fig. 1 Physicians and other EHR user groups reporting frequent EHR use in four clinical scenarios. Note: Baseline time period refers to 1 month prior to the transition to a commercial EHR. Other time periods refer to months after the transition. For example, "1 Month" refers to 1 month after the commercial EHR transition date. Other clinical EHR users included users identifying clinical care roles (e.g., respiratory therapist, radiology technician). The question stem for all survey items was: "With what frequency do you enter data into the EHR (including dictation) at the following times and locations?" The four scenarios were described on the survey as such: "On clinical workdays - in patient rooms with the patient," "On clinical workdays - between patients," "On clinical workdays - evening/nights," and "On my days off (weekdays or weekends) 


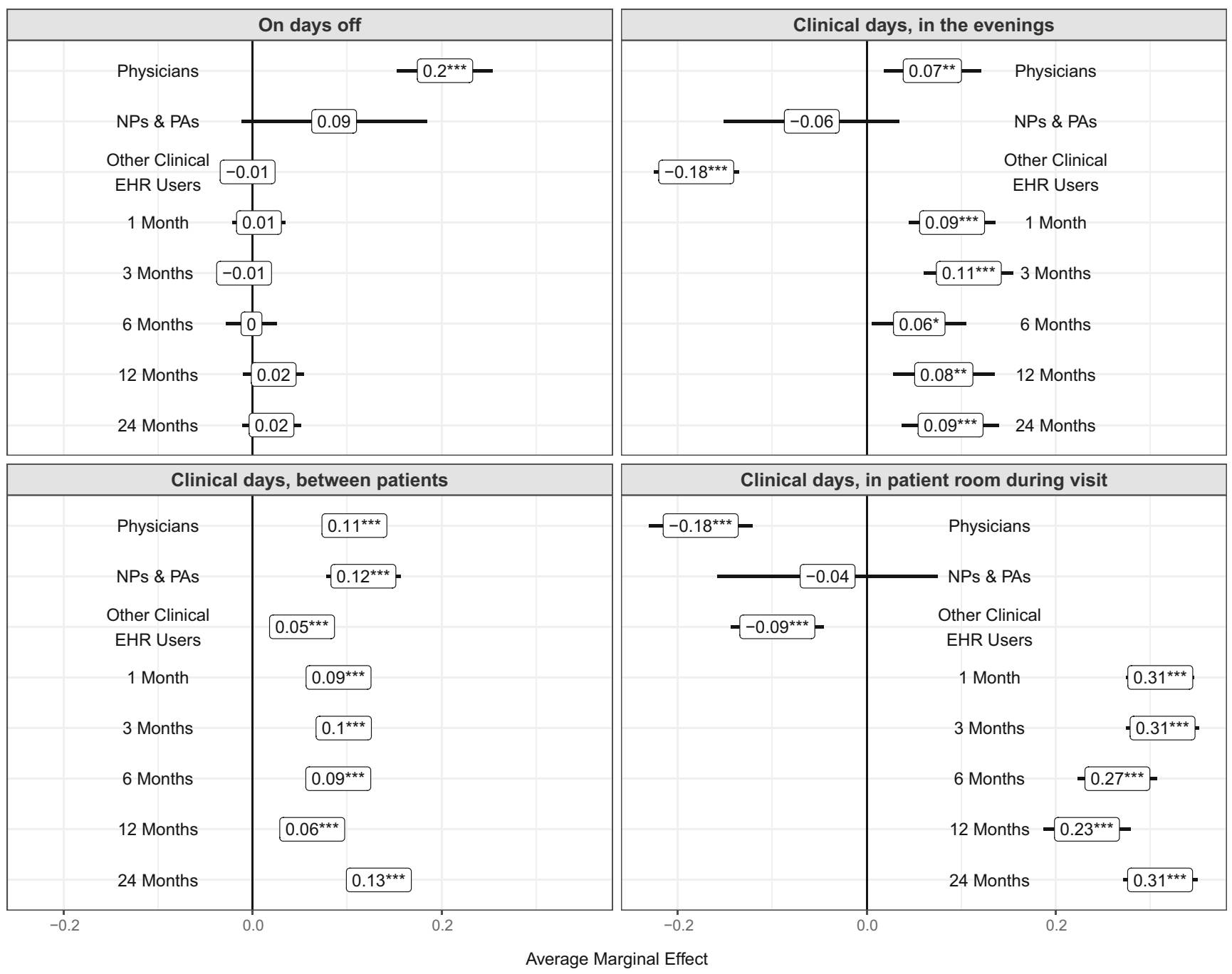

Fig. 2 Logistic regression results, marginal effects, and $95 \%$ CI. Note: Estimates can be interpreted as the percentage point change in probability of reporting frequent use in the given scenario, relative to the reference group. Reference group for all time variables is baseline survey, 1 month prior to EHR transition. Reference group for physicians, NPs and PAs, and other clinical EHR users is nurses. * $p<0.05$, $* * p<0.01, * * * p<0.001$

confounding (e.g., workflow or hardware changes). Thus, we cannot draw causal conclusions from this observational analysis. Second, our analysis does not differentiate between inpatient and outpatient providers. Third, our measures of selfreported use are inherently subjective; respondents may vary in their perceptions of "often" or "all of the time." Furthermore, we are unable to quantify this within-response variation, as respondents did not provide time estimates. Finally, we did not define hours for nights or evenings, making this interpretation subjective as well. Future studies should explore the interplay between off-hours documentation, burnout, and job satisfaction.

Nate C. Apathy, $\mathrm{Ph} \mathrm{D}^{1}$

Christopher A. Harle, $\mathrm{PhD}, \mathrm{MS}^{2,3}$

Joshua R. Vest, PhD, MPH ${ }^{4}$

Justin Morea, DO, MS, MBA ${ }^{5}$

Nir Menachemi, $\mathrm{PhD}, \mathrm{MPH}^{4}$
${ }^{1}$ Perelman School of Medicine, University of Pennsylvania,

Philadelphia, PA, USA

${ }^{2}$ Department of Health Outcomes and Biomedical Informatics, College of Medicine, University of Florida,

Gainesville, FL, USA

${ }^{3}$ University of Florida Health,

Jacksonville, FL, USA

${ }^{4}$ Richard M. Fairbanks School of Public Health, Department of Health Policy \& Management, Indiana University,

Indianapolis, IN, USA

${ }^{5}$ Eskenazi Health,

Indianapolis, IN, USA

Corresponding Author: Nate C. Apathy, PhD; Perelman School of Medicine, University of Pennsylvania, Philadelphia, PA, USA (e-mail:natea@wharton.upenn.edu). 
Funding information This work was financially supported in part by the National Library of Medicine of the National Institutes of Health under award number T15LMO12502.

\section{Compliance with Ethical Standards:}

Conflict of Interest: Mr. Apathy has nothing to disclose. Dr. Vest has nothing to disclose. Dr. Menachemi has nothing to disclose. Dr. Morea has nothing to disclose. Dr. Harle reports personal fees from Indiana Health Information Exchange, personal fees from New York eHealth Collaborative, personal fees from RTI International, outside the submitted work.

\section{REFERENCES}

1. Sinsky C, Colligan L, Li L, et al. Allocation of Physician Time in Ambulatory Practice: A Time and Motion Study in 4 Specialties. Ann Intern Med. 2016;165(11):753-760. https://doi.org/10.7326/M16-0961

2. Tai-Seale M, Olson CW, Li J, et al. Electronic Health Record Logs Indicate That Physicians Split Time Evenly Between Seeing Patients And Desktop
Medicine. Health Aff . 2017;36(4):655-662. https://doi.org/10.1377/ hlthaff.2016.0811

3. Arndt BG, Beasley JW, Watkinson MD, et al. Tethered to the EHR: Primary Care Physician Workload Assessment Using EHR Event Log Data and Time-Motion Observations. Ann Fam Med. 2017;15(5):419-426. https://doi.org/10.1370/afm.2121

4. Kroth PJ, Morioka-Douglas N, Veres S, et al. Association of Electronic Health Record Design and Use Factors With Clinician Stress and Burnout. JAMA Netw Open. 2019;2(8):e199609. https://doi.org/10.1001/ jamanetworkopen.2019.9609

5. Gardner RL, Cooper E, Haskell J, et al. Physician stress and burnout: the impact of health information technology. J Am Med Inform Assoc. 2019;26(2):106-114. https://doi.org/10.1093/jamia/ocy 145

6. Shanafelt TD, Dyrbye LN, Sinsky C, et al. Relationship Between Clerical Burden and Characteristics of the Electronic Environment With Physician Burnout and Professional Satisfaction. Mayo Clin Proc. 2016;91(7):836848. https://doi.org/10.1016/j.mayocp.2016.05.007

Publisher's Note: Springer Nature remains neutral with regard to jurisdictional claims in published maps and institutional affiliations. 